\title{
La ACTIVA PARTICIPACIÓN DE LA FACULTAD DE DERECHO DE LA UNIVERSIDAD DE LIMA EN LA LEGISLACIÓN COMERCIAL PERUANA EN LOS ÚLTIMOS CUARENTA AÑOS
}

\author{
OSWALDO HundSKOpF EXEBIO* \\ Universidad de Lima, Lima, Perú \\ Recibido: 12/11/2020 Aprobado: 19/11/2020 \\ doi: https://doi.org/10.26439/iusetpraxis2021.n053.4952
}

\begin{abstract}
RESUMEN. En este artículo, se pone de relieve la activa participación de la Facultad de Derecho de la Universidad de Lima en la gestación de importantes hitos en materia de derecho comercial peruano, así como en los cambios legislativos más relevantes de las últimas décadas en áreas tales como el derecho concursal, de protección al consumidor, arbitraje comercial, entre otros. En ese sentido, primero, se hace un breve recuento sobre el nacimiento de la Universidad de Lima y la posterior creación de su Facultad de Derecho. Seguidamente, el autor comenta su inserción en la cátedra universitaria y su labor de gestión administrativa en dicha facultad, además de la participación de diversos juristas de gran renombre que contribuyeron a la creación y consolidación de diversas cátedras y cursos del currículo de la facultad de especial contemporaneidad y relevancia académica. Finalmente, se ilustran los principales cambios normativos que tuvieron lugar en las décadas recientes en materias legislativas vinculadas al derecho comercial, en relación con las problemáticas que se buscaron atender con aquellas modificaciones y los nuevos paradigmas que estas involucraron.
\end{abstract}

PALABRAS CLAVE: Universidad de Lima / Facultad de Derecho / participación activa / cambios

\footnotetext{
*Abogado por la Pontificia Universidad Católica del Perú. Magíster y doctor en Derecho. Socio fundador en Sparrow, Hundskopf \& Villanueva Abogados Sociedad Civil de Responsabilidad Limitada. Exdecano de la Facultad de Derecho de la Universidad de Lima. Árbitro de la Cámara de Comercio de Lima, del Centro de Análisis y Resolución de Conflictos de la Pontificia Universidad Católica del Perú, y del Centro de Arbitraje de la Cámara Americana de Comercio, entre otros.
} 


\section{THE ACTIVE PARTICIPATION OF THE LAW SCHOOL OF THE UNIVERSITY OF LIMA IN PERUVIAN COMMERCIAL LAW IN THE LAST FORTY YEARS}

ABSTRACT. The author highlights the active participation of the Law School of the University of Lima in the gestation of essential milestones in Peruvian commercial law and the most relevant legislative changes in recent decades in areas such as bankruptcy law, consumer protection, commercial arbitration, among others.

The author begins his journey with a brief account of the birth of the University of Lima and the subsequent start of the Law School of said institution. Next, he comments on his insertion in the university chair and his administrative management work in said faculty, as well as the participation of various renowned jurists who passed through it and who contributed to the creation and consolidation of various chairs and courses of the faculty's curriculum of special contemporaneity and academic relevance. Finally, he illustrates the main normative changes that took place in recent decades in legislative matters related to commercial law, commenting on the problems addressed with those modifications and the new paradigms that they involved.

KEYWORDS: University of Lima / Law School / participation / active / changes 
Consideramos pertinente y necesario iniciar este artículo con una breve reseña histórica de la Universidad de Lima, con algunas notas introductorias complementarias.

Entre los años 1960 y 1961, un grupo de profesores universitarios e importantes representantes del comercio y la industria, reunidos en la Asociación Civil Prodies (Promoción del Desarrollo Industrial a través de la Educación Superior), decidieron fundar una nueva universidad. Después de dos años de esfuerzos, se creó la Universidad de Lima, cuyo funcionamiento fue autorizado por el Decreto Supremo 23 del 25 de abril de 1962.

El objetivo de Prodies era constituir una universidad diferente, acorde con el proceso de modernización del país, en la que permanentemente se fomentara el espíritu comunitario entre profesores y alumnos. Un miembro destacado de esta asociación y primer rector de la Universidad de Lima fue el doctor Antonio Pinilla Sánchez-Concha.

La Universidad de Lima inició sus labores con 120 alumnos agrupados en dos facultades, en un pequeño local situado en el distrito de Jesús María, jirón Nazca 548, frente al Campo de Marte. Debido a su rápido crecimiento, el 27 de agosto de 1966 se inauguró el campus de Monterrico con una extensión de alrededor de 40000 metros cuadrados, que hoy llega, según se nos ha informado, a 183 307,06 metros cuadrados de área utilizable.

Tenemos entendido que actualmente nuestro campus cuenta con más de 2200 lugares de estacionamiento, junto con algunos otros importantes ambientes, como el Auditorio ZUM y la Zona de Expresión Artística, los cuales son construcciones subterráneas que permiten ganar cuantioso espacio para áreas verdes en la superficie. La Universidad de Lima posee, además, a poca distancia, un campus complementario ubicado en la urbanización Mayorazgo del distrito de Ate.

En la actualidad, la Universidad de Lima tiene aproximadamente 20000 alumnos distribuidos entre su Programa de Estudios Generales, sus 12 carreras pertenecientes a 5 facultades, y su Escuela de Posgrado. Además, promueve un Instituto de Investigación Científica (IDIC) y ofrece numerosos servicios a los estudiantes, docentes y comunidad en general.

Es sumamente importante destacar que la Universidad de Lima es una institución académica sin fines de lucro, lo que la diferencia de otras universidades privadas que están constituidas y organizadas como sociedades anónimas. Sustentada en su naturaleza jurídica, forma profesionales líderes, reconocidos por sus méritos y comprometidos con el bienestar de la sociedad. Los logros de los profesionales graduados en la Universidad de Lima se constatan y comprueban en los procesos de calidad que realizan, en el desarrollo de las investigaciones y en la práctica de la innovación. La historia nos ha demostrado que en la institución se han formado excelentes profesionales de diversas carreras, que son inclusive referentes internacionales por su calidad 
académica, por el impacto de sus investigaciones y por su contribución al desarrollo integral de la sociedad. En cuanto a los valores fundamentales de la Universidad de Lima, estos son los siguientes: (a) libertad de pensamiento y respeto a la diversidad, (b) honestidad y vocación de servicio, (c) compromiso con la calidad y con la innovación, (d) lealtad y sentido de pertenencia.

La Facultad de Derecho de la Universidad de Lima, que hoy, sin duda alguna, es reconocida y muy respetada, fue creada por Resolución del Consejo Universitario 067 del 2 de octubre de 1980 como un Programa Académico de Derecho y Ciencias Políticas (así se les denominaba a las facultades en esa época, por la normativa universitaria vigente), e inició sus actividades en el mes de marzo de 1981 en el campus universitario de Monterrico. Ya funcionaban, y también destacaban en esa época, los programas académicos (después denominados facultades) de Administración, Economía, Contabilidad, Ingeniería Industrial y Comunicaciones.

En ese tiempo, se distinguía en el sistema universitario público la Universidad Nacional Mayor de San Marcos y, en el sistema universitario privado, la Pontificia Universidad Católica del Perú (PUCP), creada en 1917. La gestación y desarrollo de la Universidad de Lima desde que se fundó en 1962, además de ser una excelente idea, fue en nuestra opinión muy positiva, ya que ofrecía una nueva opción o alternativa para libremente postular a una universidad privada con una modernidad en la educación universitaria que en esa época no se ofrecía. De ser admitido como estudiante, luego de aprobar el examen de ingreso, o el traslado en su caso, se podía obtener con el esfuerzo necesario, primero, un bachillerato, luego un título profesional y, de proponérselo, una maestría como grado académico especializado.

Desde su fundación, la Universidad de Lima estuvo y está permanentemente preocupada por dirigir sus esfuerzos de educación e investigación hacia nuevas áreas, y por ello, al ingresar al mundo de las ciencias jurídicas con la creación de la Facultad de Derecho, incursionó de una manera especial y diferente hacia una perspectiva económica y política de la carrera, sobre la base de que el derecho no puede estar desvinculado de la realidad, más aún si se tiene en cuenta que en esa época venían adquiriendo singular importancia nuevos fenómenos económicos y comerciales que condujeron a la creación de instituciones innovadoras, y ya se reconocían y aceptaban alternativas o nuevas modalidades de inversión y de actividad empresarial en los diferentes sectores de la realidad. Esta coyuntura fue advertida, sostenida y difundida por las autoridades de esos primeros años de funcionamiento de la Facultad de Derecho, y me estoy refiriendo de manera especial a los doctores Camilo Carrillo, Carlos Torres y Torres Lara y Álvaro Llona Bernal.

Como sin duda es indiscutible e irrebatible, actualmente el derecho no puede estar desvinculado de la realidad, por el hecho de haber adquirido una singular relación, 
vinculación y conexidad con los fenómenos económicos y comerciales. Nunca antes la microeconomía y la macroeconomía habían tenido tanta importancia para el derecho, y ello no significa que otros campos hayan perdido importancia para las ciencias jurídicas, como la familia, los derechos sucesorios, los derechos reales y obligacionales, las relaciones contractuales, la sociedad civil en general, el derecho penal y el derecho constitucional, entre tantos otros. Lo que ocurre es que nuevos fenómenos económicos, comerciales y sociales ya habían crecido desproporcionadamente o estaban en pleno proceso de desarrollo. Así, las empresas y toda su problemática ocupaban, quiérase o no, una particular dimensión en la realidad jurídica mundial. Por ejemplo, al "comercializar" el Código italiano al derecho civil, o al emprender el Código de Obligaciones suizo la tarea de unificar las obligaciones, no han hecho otra cosa que mostrarnos las grandes características y dimensiones que está asumiendo el derecho en el mundo moderno.

A manera de comentario personalizado y con las excusas correspondientes, puesto que de lo que se trata es de destacar en forma genérica la activa participación que ha tenido la Facultad de Derecho de la Universidad de Lima en la legislación comercial peruana de los últimos cuarenta años, tal y como lo señala el título del presente artículo, no podría dejar de comentar la imprevista y no planificada incorporación del suscrito como persona y abogado al cuerpo docente, al contacto permanente con estudiantes universitarios y al grupo humano de autoridades, que tuve la inmensa fortuna de conocer, a mediados del mes de septiembre de 1974, lo que prácticamente me cambió la vida, para bien naturalmente. Mi ejercicio profesional como abogado titulado en la Pontificia Universidad Católica del Perú oficialmente se había iniciado el 20 de octubre de 1973 , fecha en la que me confirieron el título de abogado, aunque ya había laborado como practicante de derecho en distintas oficinas legales y en estudios de abogados desde el mes de enero de 1968, luego de haber concluido los dos años de Estudios Generales en la PUCP, y por haber ingresado ya al primer año de la Facultad de Derecho en el año 1968. Como recuerdo, se aconsejaba en esa época que los estudiantes de derecho complementáramos voluntariamente los estudios teóricos con las prácticas preprofesionales, combinación que, conforme aprecié y constaté, fue muy importante.

Afortunadamente para el suscrito, y la verdad sin esperarlo ni planificarlo, sucedió que a mediados del mes de septiembre de 1974, siendo ya abogado titulado, prácticamente a la mitad del segundo semestre de dicho año, el reconocido y muy apreciado profesor del curso de derecho comercial que se dictaba en la Facultad de Administración de la Universidad de Lima, el doctor Carlos Neuhaus Rizo-Patrón, inesperadamente tuvo que dejar el dictado del curso por una severa afección a la faringe y, por lo tanto, con suma urgencia se necesitaba encontrar a un reemplazante, con la condición de que se adapte al horario nocturno, ya establecido, de dos noches a la semana (miércoles y viernes de 7 p. m. a 9 p. m.). Resulta que ese año yo estaba destacado y becado por la Organización de Luis Banchero Rossi, donde trabajaba ya como parte integrante del cuerpo legal, y 
seguía, además, el curso anual de Gestión y Promoción de Exportaciones en la Escuela de Administración de Negocios ESAN para obtener el Certificado de Experto en Comercio Exterior. Entonces uno de mis compañeros de clases, cuyo padre era el doctor Sixtilio Dalmau, que también era un reconocido profesional que estaba estrechamente vinculado a la Universidad de Lima, me preguntó si yo ya era abogado graduado (en ese momento tenía 25 años de edad y hacía casi un año que ya era abogado titulado) y si aceptaría asumir a medio ciclo dicho curso, para con ello superar la emergencia suscitada por la enfermedad del doctor Neuhaus; si aceptaba, implicaba ingresar al profesorado de la Universidad de Lima para reemplazar oficialmente al profesor delicado de salud. Esta propuesta fue absolutamente inesperada, no planificada ni soñada, y me pareció muy interesante, además de que el horario de clases no alteraba mis estudios de posgrado, por lo que la acepté de inmediato y sin vacilaciones. Al respecto, considero que en esas circunstancias se me presentó una interesantísima oportunidad que de ninguna manera debía dejar pasar, y se convirtió en un gran reto personal, ya que hasta esa fecha no tenía ninguna experiencia como docente. Esta decisión me permitió integrar la planilla de profesores de la Universidad de Lima, primero en la Facultad de Administración entre los años 1974 y 1981, y luego en la Facultad de Derecho, a partir de 1981, hasta que me jubilé el 3 de febrero del 2019 al cumplir el límite de edad estatutario. Ejercí la docencia de manera ininterrumpida en la Universidad de Lima por casi 46 años, por varios años fui miembro del Consejo Directivo de la Facultad, jefe del departamento en dos oportunidades y luego decano de la Facultad de Derecho en dos periodos consecutivos.

En la segunda mitad de la década de 1970, prácticamente en medio de un gobierno militar que se había iniciado en 1968 y que concluyó en julio de 1980, éramos en realidad muy pocos los profesores que enseñábamos los cursos de derecho o los cursos vinculados a esta disciplina en la Facultad de Administración, pero todos teníamos que estar muy atentos a la evolución política y a la gestación de la nueva Constitución que se venía trabajando y que, finalmente, se aprobó en 1979. Recuerdo en esos años, entre otros, a los profesores Álvaro Llona Bernal, Jaime Zavala Costa, Fernando Orbegoso Baraybar, Ernesto Blume Fortini, Carlos Hamann Pastorino y de manera especial al doctor Carlos Torres y Torres Lara, quien tenía a su cargo los cursos de cooperativismo y de propiedad social, los cuales los dictaba bajo un enfoque básicamente jurídico. Desde que lo conocí, a pesar de ser algunos años mayor que yo, me permitió desarrollar una fraterna amistad, pues teníamos en común el hecho de haber sido ambos formados en el colegio La Salle y también en la Facultad de Derecho de la Pontificia Universidad Católica del Perú, en la que el doctor Carlos Torres y Torres Lara estudió los primeros años de la carrera de Derecho para luego trasladarse a la Universidad Nacional Mayor de San Marcos. Nos unía, además, nuestra inclinación hacia el derecho de la empresa en todas sus diferentes manifestaciones. Gracias a esa relación, pude estar al tanto de las gestiones y dificultades que se presentaron durante el proceso de gestación y creación de la Facultad de 
Derecho dentro de la Universidad de Lima, la que, como ya he resaltado, desde que había iniciado sus actividades en el año 1962, se distinguía por su perfil básicamente empresarial y, por lo tanto, una Facultad de Derecho necesariamente debía adaptarse a esta característica. Tengo entendido que no fue tarea fácil, que la idea pasó por algunas dificultades, pero que al final se formalizó con la Resolución 067 del 2 de octubre de 1980 y la Facultad de Derecho inició sus actividades en el mes de marzo de 1981. El doctor Carlos Torres y Torres Lara tuvo una importante y muy significativa participación en su gestación y desarrollo. Fue decano en dos oportunidades entre los años 1984 y 1990, en las que realizó una gestión que hasta ahora es reconocida y destacada por todos quienes estamos vinculados a la Universidad de Lima.

Como una muy especial deferencia al doctor Carlos Torres y Torres Lara, quien falleció a los 57 años el 16 de junio del año 2000, recibí el honroso encargo de presidir la comisión editora de su libro homenaje, publicado en junio del 2019, y de hacer la presentación de este. En un apretado resumen de este encargo, y con el fin de ponderar algunos conceptos, ideas y actividades de su persona, consideré oportuno y pertinente identificar tres diferentes periodos de su vida, a saber: el primer periodo es esencialmente académico y universitario, estrictamente vinculado a la Universidad de Lima, que concluyó parcialmente el 28 de julio de 1990 cuando juramentó como ministro de Trabajo; el segundo periodo abarca su trayectoria como político y culmina con su fallecimiento en el año 2000, aunque, en los últimos años, ya se había reincorporado a la docencia tanto en la Facultad de Derecho como en la Escuela de Posgrado de la Universidad de Lima; y, finalmente, un tercer periodo que se expresa a través de sus obras y las publicaciones que en su memoria se han hecho, en las que con justicia se destacan y recuerdan sus calidades humanas, profesionales, académicas y políticas.

Dentro del primer periodo, debo destacar la fuerza que le dio, en su calidad de decano, a la renovación y modernización del plan de estudios de la Facultad de Derecho de la Universidad de Lima, incorporando al cuerpo de profesores, en una primera etapa, a los doctores José León Barandiarán, Ulises Montoya Manfredi, Luis Alberto Sánchez, Francisco Miró Quesada Cantuarias, Carlos Fernández Sessarego, Valentín Paniagua, Fernando Vidal Ramírez, Felipe Osterling Parodi, Max Arias-Schreiber, Domingo García Rada y Raúl Ferrero Costa, entre otros; y, posteriormente, a Augusto Ferrero Costa y a José León Barandiarán Hart, quienes también llegaron a ser decanos de la facultad; y a los doctores Enrique Elías Laroza, Hernando Montoya Alberti, Ricardo Beaumont Callirgos, Víctor García Toma y Delia Revoredo Marsano, entre otros. En el currículo de estudios de la Facultad de Derecho ya se distinguía un importante grupo de cursos esenciales básicos y obligatorios, y una interesante variedad de cursos electivos de diferentes especialidades, entre los cuales se incluyeron cursos modernos relacionados con el derecho comercial, hoy identificado como derecho empresarial, y la informática jurídica. 
Considero de suma importancia destacar que un reducido grupo de profesores, a su solicitud, acompañamos al doctor Carlos Torres y Torres Lara a visitar en varias ocasiones a los miembros que aún se reunían para laborar con la comisión creada por el Decreto Supremo 95 del 1 de marzo de 1965, encargada de preparar un anteproyecto de un nuevo Código Civil. Esta comisión, que venía trabajando lenta y pausadamente desde el mes de marzo de 1965, había intensificado y acelerado su labor a partir del 28 de julio de 1980, cuando terminó el gobierno militar y retornó a la política el arquitecto Fernando Belaunde Terry como presidente de la República, quien designó al doctor Felipe Osterling Parodi como el primer ministro de Justicia'. El doctor Osterling Parodi cambió la metodología de trabajo de la comisión encargada de elaborar el anteproyecto del Código Civil: se redistribuyeron los temas y especialidades, y se incorporó a jóvenes y brillantes abogados, entre los cuales destacaban Lucrecia Maisch von Humboldt, Delia Revoredo Marsano, Jorge Vega Velasco y Carlos Cárdenas Quiroz, coincidentemente todos ellos ya profesores de la Facultad de Derecho de la Universidad de Lima, como también lo eran los doctores Osterling Parodi y Arias-Schreiber. Se apreciaba sin duda que había un decidido propósito de aprobar lo más rápido posible un nuevo Código Civil que reemplazara al vetusto Código de 1936, lo que se materializó el 24 de julio de 1984, fecha en la que se aprobó el Decreto Legislativo 295, mediante el cual se promulgó el Código Civil de 1984 actualmente vigente, disponiendo que este regiría a partir del 14 de noviembre de 1984.

En esos años tan especiales para el derecho civil (entre julio de 1980 y julio de 1984), eran dos los aportes o propuestas que, a criterio del doctor Torres y Torres Lara y del mencionado grupo de profesores que tuve el honor de integrar, considerábamos que se debían incorporar de una manera clara y transparente en el articulado del nuevo Código Civil. En primer lugar, la unificación de la teoría del acto jurídico y de las obligaciones civiles y comerciales, lo cual se logró parcialmente. En segundo lugar, la introducción de regulaciones específicas de contratos especiales de naturaleza comercial, cuyos proyectos veníamos trabajando. Se trataba de algunos contratos modernos que bien merecían ser recogidos en el libro de contratos del Código Civil, en razón de que ya sabíamos que en el anteproyecto se habían incluido contratos de naturaleza comercial, como son el contrato de suministro y el contrato de hospedaje, y fue por ello que presentamos los mencionados proyectos, los cuales tenían que ver con el fenómeno moderno de la distribución comercial, como son el contrato de agencia, el contrato de distribución comercial, el contrato de concesión privada y el contrato de franquicia o franchising. Lamentablemente, recibimos como respuesta que el anteproyecto ya estaba concluido

1 El Ministerio de Justicia había desaparecido durante el Gobierno Revolucionario de las Fuerzas Armadas, por lo que el doctor Felipe Osterling Parodi tuvo que refundar este ministerio. A él le sucedieron como ministros de Justicia Enrique Elías Laroza, Armando Buendía, Max AriasSchreiber y Alberto Musso, por un año cada uno, tal y conforme se había acordado en la alianza entre los partidos políticos Acción Popular y Partido Popular Cristiano. 
y que era imposible ampliar el libro VII en el que se regulan los contratos nominados, pues la comisión encargada ya había presentado oficialmente su anteproyecto y tan solo faltaba la revisión por la Comisión Revisora. Hacer cambios implicaba la reinstalación de la primera comisión, lo cual era imposible.

Siempre dentro del primer periodo de la vida del doctor Carlos Torres y Torres Lara, no se puede dejar de señalar que, en el año 1987, publicó el libro Derecho de la empresa, prologado nada menos que por el eminente jurista Francesco Galgano, profesor principal de la Universidad de Boloña, Italia, y uno de los mejores juristas de la época. El citado libro contiene dos partes. En la primera, relativa a la moderna teoría de la empresa, desarrolla el contenido y justifica la consolidación del denominado derecho de la empresa, pues este se constituye como un elemento esencial y unificador del derecho civil y del derecho comercial; incluye, además, un interesante análisis del régimen constitucional de la empresa en la Constitución Política de 1979. Por primera vez en nuestro país, un profesor peruano investigó la doctrina extranjera más importante y estructuró una teoría de la empresa en la que confluye el aporte de diversos campos, tales como el constitucional, el civil, el comercial, el laboral, el tributario, el administrativo y las ciencias políticas, lo que significó un enfoque moderno de la empresa. En la segunda parte del libro, su autor compila un material sumamente valioso para la comprensión del fenómeno empresarial: se trata de textos legales de países donde la empresa tiene ya un tratamiento especial, así como una selección de las ponencias y debates que sobre la empresa se dieron durante la elaboración de la Constitución de 1979.

Posteriormente, en el año 1989, el doctor Carlos Torres y Torres Lara publicó un segundo volumen sobre el derecho de la empresa que incluyó nuevos temas que realmente merecen destacarse, no solamente por su novedad, sino también por sus implicancias prácticas. En este libro se abordaron temas relacionados con la quiebra y reflotamiento de las empresas, el balance falso como delito de empresa, la actividad empresarial del Estado y el régimen constitucional en el área andina, entre otros. Debo resaltar que de manera excepcional, por primera vez, un autor peruano investigó y desarrolló el tema de la empresa y la familia; es decir, analizó el concepto de patrimonio familiar empresarial, los problemas prácticos que ya se suscitaban en nuestro país, y los modelos de desarrollo y de la estructura legal de la manera como debían organizarse las pequeñas empresas familiares.

Ingresando ya a una labor conjunta de todos los que fuimos autoridades y docentes de la Facultad de Derecho en la década de 1990, fuimos conscientes de que tanto la Universidad de Lima como nuestra facultad son en esencia instituciones al servicio de la comunidad y que, por lo tanto, debían proyectarse al servicio de la sociedad y en especial al ámbito jurídico. Así se inició una intensa actividad de extensión dirigida a los profesionales, profesores y alumnos de la comunidad en general, y se organizaron y llevaron a cabo eventos de gran nivel que detallamos a continuación: 
- El Congreso Internacional sobre el Código Civil Peruano, con la participación de distinguidos juristas de Argentina, Brasil, Chile, Colombia, España, Italia, México, Venezuela y Perú.

- El Primer Fórum de Derechos Humanos, organizado conjuntamente con el Instituto Interamericano de Derechos Humanos.

- El Fórum de Derecho Empresarial, que contó con la presencia del reconocido profesor de la Universidad de Boloña, doctor Francesco Galgano.

- El Congreso de Derecho Constitucional, organizado conjuntamente con el Centro de Estudios Legislativo-Económicos y Sociales (Celes), al que asistieron destacados constitucionalistas de Argentina, España y Perú.

- El Fórum sobre la Teoría Tridimensional del Derecho, para el cual se invitó al propio gestor de dicha teoría, el doctor Miguel Reale, jurista brasileño.

- El Ciclo de Conferencias sobre Derecho, Economía y Propiedad, a cargo del jurista, decano en este entonces, de la Facultad de Economía de la Universidad de Nápoles, doctor Francesco Lucarelli.

Sin duda alguna, la disciplina jurídica en la que es muy fácil demostrar la activa participación de la Facultad de Derecho de la Universidad de Lima en la legislación peruana es el derecho comercial, pues quienes éramos profesores de las materias que lo integran también fuimos promotores, propulsores e impulsadores de los cambios que se necesitaban con urgencia en derecho societario, en títulos valores, en la regulación del arbitraje como mecanismo alternativo de solución de controversias, en la tutela del consumidor, en el derecho concursal y en el derecho de seguros, entre otras materias. Asimismo, estos eran cursos que los estudiantes requerían, esperaban y en los que se matriculaban con especial interés, producto de las propuestas y debates que en esa época se hacían públicos.

En ese sentido, vamos a seguir el orden que nos hemos fijado, y por ello comenzaremos con el gran cambio que vivimos con la gestión y aprobación de la Ley 26887, Ley General de Sociedades (en adelante, LGS), que entró en vigencia el 1 de enero de 1998. En la tercera disposición final de esta ley, se derogó la Ley 16123, modificada por el Decreto Legislativo 311 y todas sus ampliatorias, derogatorias y modificaciones posteriores; el Decreto Legislativo 672; los artículos 260 al 268 del Decreto Legislativo 755, así como las leyes y demás disposiciones que se opongan a la LGS. A pesar de ello, no deberíamos dejar de comentar su antecedente, la Ley 16123 del año 1966, que tuvo como principal virtud la modificación de las secciones primera y segunda del libro segundo del Código de Comercio del año 1902, con el cual se regulaba el contrato de sociedad de naturaleza básicamente comercial. La promulgación del Código Civil de 1984, mediante Decreto Legislativo 295, trajo como consecuencia la necesidad de adecuar nuestro ordenamiento 
jurídico al cuerpo de normas y principios recogidos en tan importante norma legal. Debido a su nueva técnica legislativa, las sociedades civiles se desprendieron del Código Civil de 1984 y, con arreglo al Decreto Legislativo 311 de 1984, su normativa se incorporó en una nueva Ley de Sociedades Mercantiles, que sustituyó su denominación por Ley General de Sociedades, sin que ello implique un cambio sustantivo en la regulación legal de cada una de las sociedades allí incluidas.

En efecto, las sociedades originalmente reguladas por la Ley 16123 no perdieron su carácter mercantil, que es sinónimo o equivalente de comercial, y el legislador en el Decreto Legislativo 311 únicamente se limitó a introducir leves modificaciones esquemáticas en los artículos que componían sus disposiciones. En cuanto a las sociedades civiles, su inclusión en la Ley General de Sociedades obedeció sobre todo a un criterio de integración en un solo cuerpo legal de las formas societarias reconocidas por la ley, sin que ello supusiera comercialización o mercantilización. Antes bien, el legislador se cuidó de mantener los lineamientos fundamentales bajo los cuales estaba regulada la sociedad civil en el Código Civil de 1936; incluso llegó a repetir casi textualmente algunos de sus artículos.

Teniendo en cuenta que mediante el Decreto Legislativo 311, Ley de Sociedades Mercantiles, se había modificado la Ley 16123 para integrar en un solo texto a las sociedades civiles y mercantiles, por razones estrictamente formales y de orden, fue necesario que por el Decreto Supremo 003-85-JUS, del 14 de enero de 1985, se apruebe un texto único ordenado de la Ley General de Sociedades, que reguló por primera vez en un solo cuerpo legal a ambos tipos de sociedades.

Entre 1985 y 1995, en razón de la propia dinámica del derecho societario -que está en permanente modernización y cambio-, así como por las innovaciones introducidas en esos años en nuestro ordenamiento jurídico, y por la conveniencia de adecuar la legislación societaria a las nuevas concepciones y principios rectores de la economía de nuestro país (recogidos en la Constitución de 1993), con una muy activa participación de los profesores de derecho comercial de la Facultad de Derecho de la Universidad de Lima, se llevaron a cabo innumerables eventos académicos, seminarios, foros y mesas redondas, e incluso se publicaron múltiples artículos y ensayos relacionados con la necesidad o conveniencia de contar con un nuevo marco legal societario. En esos eventos se identificaron de manera clara y precisa las razones que justificaban una nueva Ley General de Sociedades, lo cual era un proceso inevitable e ininterrumpible. Tales razones fueron las siguientes:

- La necesidad de modernizar la legislación societaria y adecuarla a la nueva realidad, así como proyectarla al siglo XxI.

- En el año 1994, se contaba con una legislación societaria desordenada, dispersa y contradictoria, y sin lugar a dudas, había mucha distancia entre la realidad y la normativa societaria. 
- La legislación anterior contenía errores formales. A manera de ejemplo, en los artículos 78 (inciso 5), 107 y 130 se seguía hablando de acciones al portador, cuya existencia estaba prohibida desde el año 1968.

- Asimismo, en la legislación anterior se reconocía una excesiva importancia a la acción como título: es el caso de los artículos 111 y 112, sobre la prenda y el embargo de acciones; el del artículo 145, que exigía como recaudo para la acción de impugnación de acuerdos el previo depósito de los certificados de acciones en una institución bancaria; y el del artículo 223, sobre la reducción de capital por canje de títulos.

- Había una confusión en el uso de términos: capital social, capital suscrito y capital pagado, en relación con los derechos de los accionistas.

- Se regulaba un órgano que había demostrado su inutilidad: el Consejo de Vigilancia.

- Existían legislaciones paralelas sobre sociedades abiertas y sociedades de accionariado difundido.

- Se necesitaba incorporar nuevas instituciones, como los convenios de accionistas, la autocartera de acciones, la escisión de sociedades y otras formas de reorganización societaria.

- Se requería actualizar y modernizar las normas sobre estados financieros, balances, utilidades y repartición de dividendos.

- Se necesitaba adecuar los procedimientos judiciales en relación con el nuevo Código Procesal Civil de 1993, que ya se encontraba plenamente vigente.

- Se requería concordar la legislación societaria con el nuevo sistema de reestructuración empresarial, también conocido como sistema concursal, y con la legislación sobre el mercado de valores.

- Finalmente, se necesitaba ordenar y unificar la regulación sobre los contratos asociativos.

A manera de conclusión sobre este punto, se puede afirmar que la corriente mayoritaria, y que representaba un clamor del empresariado, era que una nueva Ley General de Sociedades acabaría con los vacíos y ambigüedades de la normativa societaria, y la reordenaría íntegramente, lo cual en efecto sucedió.

En el panorama descrito, el Ministerio de Justicia, mediante la Resolución Ministerial 424-94-JUS, del 1 de septiembre de 1994, publicada el 5 de septiembre de ese año, nombró una comisión que elabore un anteproyecto de una nueva Ley General de Sociedades, conformada por connotados profesionales especialistas en derecho comercial, y a la 
que se le otorgó inicialmente un plazo de 120 días. Posteriormente, por la Resolución Ministerial 075-95-JUS, del 27 de febrero de 1995, publicada el 2 de marzo de ese año, se prorrogó dicho plazo por 90 días adicionales; sin embargo, dada la complejidad de la tarea legislativa encomendada a la citada comisión, se consideró necesario ampliar el plazo a un año, para culminar satisfactoriamente con la redacción y formulación del referido anteproyecto. La ampliación se formalizó mediante la Resolución 270-95-JUS, de fecha 19 de julio de 1995, publicada el 20 de julio de ese año. Finalmente, y en razón de la ardua e importante tarea encomendada, la Comisión Redactora solicitó una prórroga del plazo otorgado para culminar satisfactoriamente con la redacción y formulación del anteproyecto de la Ley General de Sociedades. Dicha solicitud fue concedida mediante la Resolución Ministerial 159-96-JUS, del 12 de julio de 1996, publicada el 16 de julio de ese año, en la que se ampliaba por última vez el plazo hasta el 31 de diciembre de 1996.

La mencionada comisión cumplió con la entrega del referido anteproyecto al Ministerio de Justicia dentro del plazo concedido, el cual lo remitió al Congreso de la República, que a su vez lo derivó a la Comisión de Constitución y Reglamento del Congreso. La inicialmente denominada Comisión Elaboradora del Anteproyecto de la Ley General de Sociedades, conocida después como Comisión Redactora del Anteproyecto de la Ley General de Sociedades, estuvo integrada por once juristas vinculados al derecho comercial: Enrique Normand Sparks (quien la presidió), Enrique Elías Laroza, Francisco Moreyra García Sayán, Álvaro Llona Bernal, Alfonso Rubio Feijóo, Julio Salas Sánchez, Hernando Montoya Alberti, Ricardo Beaumont Callirgos, Alfredo Ferrero Diez-Canseco, Emil Ruppert Yáñez y por el suscrito, con lo cual es muy fácil confirmar que seis de los once miembros éramos profesores de la Facultad de Derecho de la Universidad de Lima, lo cual merece destacarse.

Asimismo, es pertinente recordar que la Comisión de Constitución y Reglamento del Congreso, encargada de estudiar el referido anteproyecto de ley, emitió el dictamen de fecha 18 de febrero de 1997 en el cual señaló que era innecesario delegar facultades al Poder Ejecutivo para que mediante decreto legislativo apruebe la Ley General de Sociedades, tal como fue solicitado por el Ejecutivo. Además, en su lugar, acordó por unanimidad proponer al pleno del Congreso que se delegue única y exclusivamente a la Comisión Permanente del Congreso de la República la facultad de legislar dentro de un plazo máximo de 180 días, en los términos a que hace referencia el artículo 101 de la Constitución, para dictar una nueva Ley General de Sociedades, sobre la base del anteproyecto elaborado por la Comisión creada mediante la Resolución Ministerial 424-94-JUS, con la recomendación de que se constituya una Comisión Revisora en la cual participasen como invitados los autores del anteproyecto de la Ley General de Sociedades, todo ello con el propósito de despolitizar el tema y lograr que se apruebe una norma legal con mayor y mejor técnica legislativa, y con un contenido de mayor impacto y efecto. 
Fue gracias a ello que el Congreso de la República, mediante la Resolución 001-97CR, del 20 de febrero de 1997, publicada el 22 de febrero de ese año, aprobó dicha delegación y, además, acordó designar al doctor Carlos Torres y Torres Lara como presidente de dicha comisión que estuvo integrada por los congresistas Jorge Muñiz Ziches, Jorge Trelles Montero, Jorge Avendaño Valdez y Javier Alva Orlandini. Posteriormente, en la sesión de instalación de la Comisión Revisora, llevada a cabo el día 5 de marzo de 1997, el presidente de la comisión propuso que se incorpore a los congresistas Lourdes Flores Nano y Ricardo Marcenaro Frers.

En lo que concierne al doctor Carlos Torres y Torres Lara, fue muy importante el rol que le tocó desempeñar como presidente de la Comisión Revisora, ya que desde el comienzo fue un convencido de que la Ley General de Sociedades, sin tener la categoría de una ley orgánica por su contenido técnico, era una ley muy importante que debía ser vista por el propio Congreso en lugar de ser aprobada por decreto legislativo, mediante una delegación de facultades al Poder Ejecutivo. En su opinión, se debía hacer uso de las facultades que otorgaba la Constitución de 1993 y, por tanto, se tenía que dar facultades extraordinarias al propio Congreso, pero a la Comisión Permanente, que fue en la práctica lo que sucedió. En otras palabras, la que es hoy la LGS fue la primera ley emitida y aprobada por la Comisión Permanente en nombre y representación del Congreso, sin que pase por el pleno. Lo mismo sucedió con la Ley 27287, Ley de Títulos Valores, a la que nos referiremos posteriormente.

De acuerdo con lo manifestado por el doctor Enrique Normand Sparks, presidente de la Comisión Redactora del Anteproyecto, en su exposición de presentación al Congreso, publicada en el diario oficial El Peruano el 23 de abril de 1997, la intención de elaborar un anteproyecto de una nueva Ley General de Sociedades obedeció a la necesidad de revisar, corregir, innovar, cambiar, modificar y complementar el antiguo texto legislativo, y hacerlo acorde con la gran transformación del marco económico del Perú en los últimos años. En el caso de la legislación mercantil en nuestro país, esta acumulaba una experiencia de más de noventa años, lo cual, dentro de la rápida dinámica del derecho societario y la evolución económica mundial, justificaba plenamente la dación de un nuevo instrumento legal. El trabajo realizado por la Comisión Redactora estuvo destinado a elaborar un texto que recogiera y comprobara fielmente la realidad nacional.

La Comisión Redactora prescindió de la labor fácil de copiar alguna de las legislaciones extranjeras, como ocurrió con el Código de Comercio de 1902 y con la anterior normativa societaria. Por un lado, se respetaron las instituciones y textos de artículos que funcionaron eficiente y claramente, y, por otro, se acudió a la consulta del derecho comparado, con preferencias por las legislaciones española, mexicana, chilena, inglesa, suiza y francesa.

Asimismo, en su trabajo, esta comisión consultó a diversas instituciones, tales como la Conasev, la Bolsa de Valores de Lima, el Colegio de Abogados, el Colegio de Notarios 
de Lima, la Cámara de Comercio, la Confiep, el Indecopi y la Asociación de Bancos. Asimismo, se contó con la asesoría de distinguidos y destacados auditores para los aspectos contables y de auditoría que contempla la ley.

También es importante señalar que la comisión estructuró un plan de trabajo para la elaboración del citado anteproyecto, y para temas puntuales y especializados, se contó con la colaboración de expertos en materias multidisciplinarias.

Igualmente, debe destacarse que al anteproyecto se le dio la máxima publicidad y divulgación, lo que no se había hecho con ninguna otra ley anterior, para que las instituciones y personas naturales o jurídicas interesadas en el tema participen con sus sugerencias y recomendaciones en los diversos aspectos abordados por dicha norma, algunas de las cuales se recogieron en el texto final. La secuencia de las publicaciones en el diario oficial El Peruano fue la siguiente:

- El 2 de marzo de 1997 se publicó el anteproyecto elaborado por la comisión designada por la Resolución Ministerial 424-94-JUS, tal y como fue presentado al Ministerio de Justicia.

- El 23 de abril de 1997 se publicó la exposición efectuada por el doctor Enrique Normand Sparks ante la Comisión Revisora del Congreso de la República, la cual complementa el anteproyecto presentado. Dicha exposición se puso a disposición de las instituciones especializadas, profesionales en derecho y ciudadanos interesados en su contenido para que la estudien y envíen sus opiniones y sugerencias a la Primera Vicepresidencia del Congreso, incluso a través de internet.

- El 10 de mayo de 1997 se publicó la versión aprobada por la Comisión Revisora del Congreso de la República, antes de ser elevada a la Comisión Permanente del Congreso.

- El 3 de octubre de 1997 se aprobó el Proyecto de Ley General de Sociedades refrendado por la Comisión Permanente, a efectos de recibir dentro de los 15 días siguientes de su publicación opiniones y sugerencias de los interesados.

- El 28 de octubre de 1997 se puso en conocimiento de la comunidad, publicándose el documento final antes de su formalización por la Comisión Permanente, y se abrió un último plazo para la recepción de sugerencias.

- Finalmente, el 9 de diciembre de 1997 concluyó la secuencia con la publicación de la LGS.

Sin lugar a dudas, los que integramos la comisión que elaboró el anteproyecto, en especial, y quienes mostraron interés en su contenido y alcances tuvimos reiteradas oportunidades para participar con opiniones, sugerencias y recomendaciones, las cuales fueron recibidas y evaluadas por la Comisión Redactora, por los congresistas y 
por sus asesores especializados; $y$, de acuerdo con su pertinencia y viabilidad, muchas de ellas se incorporaron al texto final, sin que se haya alterado la estructura y espíritu del anteproyecto.

Si bien el proceso de elaboración de la LGS fue largo, como ya lo hemos mencionado, inició su vigencia a partir del 1 de enero de 1998, derogando expresamente la Ley 16123 y todas sus disposiciones ampliatorias posteriores; y ahora está próxima a cumplir 23 años de vigencia. En dicha ley se introdujeron importantes innovaciones y cambios, adecuando y preparando nuestra estructura legal societaria frente a las vicisitudes del siglo xx que ya estaba concluyendo, y sobre todo para el siglo xxI.

Acto seguido, la Facultad de Derecho y Ciencias Políticas de la Universidad de Lima, con el apoyo de su decano en ese entonces, José León Barandiarán Hart, y de la Dirección Universitaria de Proyecto Social, así como con el auspicio de Gaceta Jurídica, organizó y llevó a cabo entre los días 27 y 29 de enero de 1998, con notable éxito de asistencia, el primer seminario de nivel académico universitario de divulgación y difusión de la LGS. Asimismo, la Facultad de Derecho de la Universidad de Lima y sus expositores (profesores nuestros vinculados al derecho comercial) nos propusimos y ofrecimos presentar por escrito cada una de las ponencias con el objeto de publicar un libro especial elaborado por el Fondo de Desarrollo Editorial de la Universidad de Lima, para contribuir a la difusión y conocimiento de la citada ley. Cumplimos con nuestra promesa y sometimos a consideración del público interesado la primera edición del libro Nuevo derecho societario (1998), respetando el mismo orden en que se dieron las exposiciones. Esta publicación tuvo indiscutible éxito de difusión y venta.

Continuando con el orden que nos hemos fijado, a continuación nos referiremos al proceso de gestación y aprobación de la Ley 27287, Ley de Títulos Valores (en adelante, LTV), publicada el 19 de junio del 2000. Quienes, como abogados, de un modo u otro estábamos vinculados al derecho comercial, ya sea en la docencia, en la asesoría legal de empresas o en la defensa ante los tribunales de justicia, éramos conscientes de que la antigua Ley de Títulos Valores, la Ley 16587 del año 1967, se estaba tornando anacrónica y rezagada con la modernidad, situación que felizmente ya se había superado en materia societaria con la aprobación de la LGS. En derecho comercial, qué duda cabe que tradicionalmente las dos leyes más importantes son la de Sociedades y la de Títulos Valores, sin desconocer tantas otras de sumo interés, algunas de las cuales también comentaremos a continuación y que son la legislación sobre la regulación del arbitraje como mecanismo alternativo de solución de controversias, la legislación sobre protección del consumidor, la legislación sobre el sistema concursal (antes reestructuración patrimonial) y la legislación sobre el contrato de seguros; reconocemos, además, la existencia de la legislación sobre el transporte marítimo, la legislación sobre la libre competencia, la legislación sobre protección de los derechos intelectuales y de propiedad industrial, y sobre el mercado bursátil. Recordamos con especial interés la década de 1990 y la 
primera década del siglo en curso, por el interés que había suscitado en el mundo jurídico peruano la nueva legislación sobre el Indecopi y sobre el mercado de valores tanto nacional como internacional.

En relación con los títulos valores, por las Resoluciones 068-97-JUS, 110-97-JUS y 161-97-JUS, se integró una comisión encargada de elaborar un proyecto de nueva Ley de Títulos Valores y se le concedió seis meses de plazo. Esta comisión estuvo presidida por el doctor Ricardo Beaumont Callirgos e integrada por los doctores Jorge Ramírez Díaz, Óscar Pomar Fonseca, Hernando Montoya Alberti, Rolando Castellares Aguilar, Gonzalo García Calderón, Alonso Morales Acosta, Juan Manuel Echevarría, Pedro Flores Polo y por el suscrito, es decir, por diez miembros, ocho de los cuales éramos profesores de la Facultad de Derecho de la Universidad de Lima (no lo eran Pomar Fonseca y Flores Polo). En mi caso, por un lamentable cruce con los horarios del Directorio de la Conasev, del cual formaba parte en esa época, tuve que renunciar y fui reemplazado por el también profesor de la Facultad de Derecho de la Universidad de Lima, Óscar Zegarra Guzmán.

Como bien comenta el doctor Ricardo Beaumont Callirgos en la introducción de la obra jurídica que elaboró conjuntamente con el doctor Rolando Castellares Aguilar, titulada Comentarios a la nueva Ley de Títulos Valores (2000), las primeras reuniones de la comisión fueron orientadas a discutir y acordar la metodología del trabajo encomendado, y a distribuir las ponencias que se debían preparar para el debate en el pleno, las cuales fueron decididas según sugerencias de los propios miembros, es decir, por su particular acercamiento académico o de investigación con algún punto en especial. Así, uno de ellos tomó en exclusiva la letra de cambio; otro prefirió tratar las garantías, tales como avales y fianzas; otro las cláusulas especiales; otro la factura conformada; y otro el título de crédito hipotecario negociable, etcétera. Para tal efecto, se hizo una relación de todos los títulos valores que tenían vigor en el Perú hacia 1997-1998, y el primer acuerdo y decisión de la comisión fue que el nuevo texto reúna a todos los papeles comerciales, evitando el desorden y confusión que había significado la ley anterior, Ley 16587 , que solo regulaba tres títulos valores: la letra de cambio, el cheque y el pagaré o vale a la orden.

Un segundo e importante acuerdo fue el de hacer referencia a que, si bien el Supremo Gobierno había pedido redactar un proyecto de nueva Ley de Títulos Valores, la comisión debía excederse del encargo y presentarle alternativamente un proyecto de nueva Ley de Valores Negociables, pues en el derecho cambiario moderno se reconoce e identifica como género a los valores negociables, y como especie (1) a los valores en título o títulos valores y (2) a los valores electrónicos, que la propia Ley del Mercado de Valores, Decreto Legislativo 861, denomina anotaciones en cuenta y registro. En efecto, los primeros constan en soporte papel, y de allí el modo de referirse a los derechos incorporados en tales documentos como derechos cartulares, derechos cartáceos, derechos documentarios, y a los principios de incorporación del derecho al título-título (tangible) 
y derecho (intangible), los cuales se confunden y, teniendo comunidad de destino, son particularmente inescindibles. Para Beaumont Callirgos y Castellares Aguilar (2000), en la introducción de la obra jurídica que hemos mencionado, era importante destacar que la Comisión Redactora no solo debía abocarse a elaborar un proyecto de Ley de Títulos Valores, sino plantear, además, una regulación legislativa de los valores virtuales, desmaterializados o electrónicos, lo que debería dar lugar a que la ley tenga como denominación nueva Ley de Valores Negociables, porque con dicho nombre quedan incluidos tanto los valores materializados en soporte papel, valores en título o títulos valores y los valores virtuales, electrónicos o desmaterializados, que la propia Ley del Mercado de Valores, como se dijo antes, denominaba anotaciones en cuenta y registro.

Otro acuerdo importante de la Comisión Redactora de la Ley de Títulos Valores fue el que dio lugar a la reflexión formulada en torno a qué mecanismo crear para no tener que aguardar años o décadas para incorporar en esta ley nuevos valores, en título o electrónicos, que el tráfico mercantil y los negocios bancarios y financieros podrían generar en el futuro. Debido a ello, estimamos que fue acertada la idea al proyectar un artículo, que finalmente resultó ser el tercero, concordante con el artículo 276 y con la quinta disposición final, mediante el cual el Congreso de la República encarga a entidades y organismos de control y supervisión - tales como la Superintendencia de Banca y Seguros, la Superintendencia de Administradoras Privadas de Fondos y Pensiones, y la Comisión Nacional Supervisora de Empresas y Valores (hoy SMV) - autorizar la creación, emisión, negociación y adquisición de valores mobiliarios e individuales por parte de las personas y empresas sujetas a su control, que inclusive podrán representar derechos patrimoniales distintos a los de participación o deuda, estableciendo sus condiciones, formalidades y demás requisitos; inclusive, aprobando los modelos y formatos estandarizados de aquellos propuestos por las Cámaras de Comercio y otras asociaciones gremiales.

En cuanto a la estructura general de la LTV, esta es la siguiente:

- Se divide en el libro primero, con la parte general, y el libro segundo, con la parte especial.

- El libro primero contiene: (1) las reglas generales aplicables a los títulos valores; (2) la circulación de los títulos; (3) las cláusulas especiales de los títulos valores; (4) las garantías (aval y fianza); (5) el pago; (6) el protesto; (7) las acciones cambiarias; (8) la prescripción y caducidad; (9) el deterioro, destrucción, extravío y sustracción de títulos valores; y (10) las normas de derecho internacional aplicables a los títulos valores.

- El libro segundo comprende: (1) la letra de cambio; (2) el pagaré; (3) la factura conformada; (4) el cheque; (5) el certificado bancario de moneda extranjera y el certificado bancario de moneda nacional; (6) el certificado de depósito y el 
warrant; (7) el título de crédito hipotecario negociable; (8) el conocimiento de embarque y la carta de porte; (9) los valores mobiliarios; y (10) los títulos valores especiales.

Sin lugar a dudas, consideramos que se promulgó una ley bien concebida y con buenos augurios de firmeza y confiabilidad. No debemos olvidar que la antigua Ley de Títulos Valores, Ley 16587, si bien hacía expresa referencia a los títulos al portador, a los títulos a la orden y a los títulos nominativos en sus artículos 23,26 y 29, sin embargo, solo regulaba los títulos a la orden, pues, como bien todos conocemos, en esa ley se trataron la letra de cambio, el cheque y el pagaré o vale a la orden, los tres o los cuatro, como quiera decirse, con certeza títulos valores a la orden. Para el doctor Ulises Montoya Manfredi, quien también fue profesor de la Facultad de Derecho de la Universidad de Lima en sus inicios, se trataba de cuatro documentos; en cambio, para el doctor Remigio Pino Carpio eran solo tres, pues según el texto mismo de la ley se regulaba al "pagaré o vale a la orden", es decir, un solo documento que podía llamarse o denominarse de cualquiera de esas formas.

Beaumont Callirgos y Castellares Aguilar (2000) destacan que actualmente tenemos numerosos ejemplos de títulos valores a la orden, pero también encontramos "títulos al portador"; tal es el caso del certificado bancario en moneda extranjera, artículos 218 a) y 219 c); y los "títulos nominativos", como, por ejemplo, las acciones de sociedades, artículo 257.1. En la Ley 16587, las acciones cambiarias se regulaban con ocasión de cada título valor; así, teníamos los artículos 122 a 128 para la letra de cambio y los artículos 174 a 178 para el cheque; en cambio, en la LTV, las acciones cambiarias se tratan en la parte general y son aplicables a todos los títulos valores en sus artículos 90 al 94 . Por último, el aval en la Ley 16587 solo se instituía para la letra de cambio, artículos 85 a 88; mientras que en la LTV las garantías (aval y fianza) son tratadas en el libro primero, parte general, aplicable a todos los títulos valores, artículos 56 al 63. De otro lado, la estructura de la LTV, además de su dimensión, horizonte y filosofía, es distinta de la Ley 16587.

Siguiendo con la secuencia predeterminada, nos corresponde referirnos a la novedosa regulación sobre el arbitraje como mecanismo de solución de controversias que se impulsó y desarrolló también en la década de 1990. Sin temor a equivocarme, puedo confirmar que la Facultad de Derecho de la Universidad de Lima fue la primera que incorporó la materia de derecho arbitral en su currículo de estudios; al principio, con un curso obligatorio general que estuvo a cargo del doctor Gonzalo García Calderón y, luego, con un segundo curso electivo de arbitrajes especiales a cargo del doctor Mario Castillo Freyre.

Reiterando lo que muchos juristas opinan, el arbitraje en el Perú se ha consolidado como un fenómeno único en América Latina, pues no existe otro país de nuestro continente donde este mecanismo alternativo a la jurisdicción ordinaria para la solución de controversias haya tenido un desarrollo tan rápido y vertiginoso. 
Es pertinente destacar que el arbitraje está reconocido como jurisdicción autónoma por la Constitución Política de 1993 cuando, al detallar los principios y derechos de la función jurisdiccional, declara que "no existe ni puede establecerse jurisdicción alguna independiente, con excepción de la militar y arbitral" (artículo 139, numeral 1). El arbitraje se presenta, a su vez, como una vía alternativa al Poder Judicial, ya que, al ser una jurisdicción independiente, los árbitros poseen la notio (la facultad de conocer el conflicto planteado), la vocatio (la facultad de ordenar la comparecencia de las partes) y la iuditio (la facultad de resolver el conflicto, poniendo solución a la controversia mediante la emisión del laudo arbitral).

La Constitución Política de 1979, cuya vigencia se inició el 28 de julio de 1980, por primera vez reconoció al arbitraje como una función jurisdiccional, pues en su artículo 233, inciso 1, le da este carácter al declarar la unidad y la exclusividad de la función jurisdiccional. Este precedente normativo es importante, pues contribuyó a su difusión y ulterior desarrollo.

Con el Código Civil de 1984, promulgado el 24 de julio de ese año y vigente desde el 14 de noviembre, se buscó reprivatizar el arbitraje, dejando de lado el procedimiento de la forma como se regulaba, de acuerdo con lo ya explicado; se reforzó su carácter de contrato o acuerdo de voluntades que constituye su base fundamental; se incluyó, por primera vez, un título sobre esta materia en el libro titulado "Fuentes de las Obligaciones" (artículos 1906 a 1922). Sin embargo, se ratificó la necesidad de una doble manifestación de voluntad por considerarse como contratos nominados.

Posteriormente, con la Ley 26572, que estuvo vigente a partir del 6 de enero de 1996, se aprobó una nueva Ley General de Arbitraje y se derogó el Decreto Ley 25935, que se había inspirado en la Ley Modelo de la Comisión de las Naciones Unidas para el Derecho Mercantil Internacional (UNCITRAL), aprobado por la Resolución 40/72 de la Asamblea General del 11 de diciembre de 1985. Con la Ley 26572 se alcanzó un estándar internacional aceptable. Lo más significativo fue la eliminación de la nefasta distinción entre la cláusula compromisoria y el compromiso arbitral, este último llamado convenio arbitral por el Decreto Ley 25935, para finalmente llegar al Decreto Legislativo 1071, promulgado el 27 de junio del 2008 y vigente desde el 1 de septiembre de ese año, que norma el arbitraje. En este decreto se ha regulado de manera adecuada la justicia arbitral, respaldada ahora con una norma jurídica que ofrece un marco legal moderno e idóneo.

Siguiendo con la secuencia predeterminada, corresponde abordar la legislación de protección de los derechos del consumidor, sobre la cual también se programó en la Facultad de Derecho de la Universidad de Lima un curso especial para la formación de los abogados, pues, como es público y notorio, cada día y a toda hora se llevan a cabo transacciones económicas en relaciones de consumo en las que participan proveedores y consumidores o usuarios para la adquisición de productos o para la contratación de 
servicios. No cabe duda alguna de que en estos tiempos el derecho del consumidor está presente en el día a día de todos nosotros y su importancia tiene rango constitucional, al disponerse en la Constitución Política de 1993, artículo 65, lo siguiente:

El Estado defiende el interés de los consumidores y usuarios. Para tal efecto garantiza el derecho a la información sobre los bienes y servicios que se encuentran a su disposición en el mercado. Asimismo, vela, en particular, por la salud y la seguridad de la población.

La regulación anterior al actual Código de Protección y Defensa del Consumidor, aprobado por la Ley 29571 y publicado el 2 de septiembre del 2010, era desordenada y dispersa, y era la siguiente:

- El Decreto Legislativo 716, Ley de Protección al Consumidor, la cual podría ser considerada en el Perú con la primera ley en materia de protección al consumidor (7 de noviembre de 1991).

- La Ley 27311, Ley de Fortalecimiento del Sistema de Protección al Consumidor (17 de julio del 2000).

- El Decreto Supremo 039-2000-ITINCI, Texto Único Ordenado de la Ley de Protección al Consumidor (11 de diciembre del 2000).

- El Decreto Legislativo 1045, Ley Complementaria del Sistema de Protección al Consumidor (26 de junio del 2008).

- El Decreto Supremo 006-2009-PCM, Texto Único Ordenado de la Ley del Sistema de Protección al Consumidor (26 de junio del 2008).

En el año 2009, la Presidencia del Consejo de Ministros creó la Comisión Consultiva encargada de elaborar el anteproyecto del Código de Consumo, que debía ser entregado a fines de septiembre de ese año para su evaluación por el Consejo de Ministros y, posteriormente, por el Congreso de la República. Esta comisión estuvo presidida por Walter Gutiérrez Camacho, quien es actualmente el defensor del pueblo, y que en esa época era profesor de la Facultad de Derecho de la Universidad de Lima y decano del Colegio de Abogados de Lima (CAL). Asimismo, se designó en esta comisión a destacados profesiones y abogados tales como Jaime Delgado, Rosario Fernández Figueroa, Mercedes García Belaunde de Hamann, Alonso Morales Acosta, Jorge Avendaño, Alfredo José Bullard González, Juan Alejandro Espinoza Espinoza, Yuri José Francisco Vega Mere, Juan Francisco Rojas Leo y el suscrito. Como se puede notar, cinco de los integrantes ya formábamos parte en esa época de la plana docente de la Facultad de Derecho de la Universidad de Lima (Rosario Fernández Figueroa, Alonso Morales Acosta, Juan Espinoza Espinoza, Juan Francisco Rojas Leo y yo), y lo que se debe destacar y reconocer es que el doctor Alonso Morales Acosta sigue siendo el titular del curso electivo que se dicta en la Facultad de Derecho sobre dicha materia y que se denomina Tutela del Consumidor. 
Cuando finalmente se dio el Código de Protección y Defensa del Consumidor, aprobado por la Ley 29571, se marcó un nuevo hito para esta materia. Se incorporaron varias novedades como el establecimiento de políticas públicas, la obligación de los proveedores de contar con el Libro de Reclamaciones, la regulación de protección del consumidor en productos o servicios específicos, como servicios públicos regulados, productos o servicios de salud, productos o servicios educativos, productos o servicios inmobiliarios o servicios financieros, entre otros.

Siguiendo con las materias estrechamente vinculadas a la Facultad de Derecho de la Universidad de Lima, nos corresponde referirnos al derecho concursal, el cual estuvo y está íntimamente relacionado con las actividades de toda Comisión de Reestructuración Patrimonial, área que forma parte de la jurisdicción del Indecopi, y cuya evolución y desarrollo también se incorporó como tema de estudio especializado en un curso electivo que se programó en el currículo de estudios de la facultad.

Actualmente, como es conocido, el objetivo de la Ley 27809, Ley General del Sistema Concursal, del año 2002, es "la recuperación del crédito mediante la regulación de procedimientos concursales que promuevan la asignación eficiente de recursos a fin de conseguir el máximo valor posible del patrimonio del deudor" (artículo I del "Título preliminar"), y cuya finalidad es "propiciar un ambiente idóneo para la negociación entre los acreedores y el deudor sometido a concurso, que les permita llegar a un acuerdo de reestructuración 0 , en su defecto, a la salida ordenada del mercado, bajo reducidos costos de transacción" (artículo II del "Título preliminar").

El Indecopi tiene su sede oficial en Lima. No obstante, en los noventa, descentralizó algunas de sus funciones en materia concursal. En efecto, mediante el Decreto Legislativo 788, publicado el 31 de diciembre de 1994, se lo declaró en reorganización para disponer su descentralización y modificar algunos artículos de la Ley de Organización y Funciones del Indecopi. Se estableció en su artículo 2 lo siguiente:

Las oficinas y comisiones de dicho Instituto, previa aprobación del Directorio, podrán delegar sus funciones, por medio de la suscripción de los convenios correspondientes, en organismos, instituciones o entidades públicas, o en las Cámaras de Comercio, Colegios Profesionales, o entidades gremiales de reconocido prestigio, siempre que se garantice la total independencia de su actuación.

En mérito a esos convenios, fue posible la recepción y distribución de la carga procesal del Indecopi entre las distintas comisiones delegadas, siempre que tengan el mismo ámbito de competencia territorial.

En lo que respecta a la materia concursal, se suscribieron sendos convenios de delegación de funciones tanto en Lima como en provincias con cámaras de comercio, universidades, entre otros. Así, en la Universidad de Lima se suscribió un convenio y por ello funcionaba una Comisión de Reestructuración Patrimonial de la Oficina 
Descentralizada del Indecopi, cuyo aporte a la sociedad fue trascendental para conocer las causas relativas a la reestructuración o liquidación de deudores en graves crisis económicas, según el marco de los requisitos legales vigentes.

Ahora bien, se hizo necesaria la dación de la Ley 27809, Ley General del Sistema Concursal (en adelante, LGSC), del año 2002, pues se observó que las anteriores normas presentaban una regulación muy difusa. Como referencia histórica, el sistema concursal, ahora así denominado, se estableció en nuestro país en 1992 con la promulgación de la denominada Ley de Reestructuración Empresarial, Ley 26116, que derogó la Ley Procesal de Quiebras vigente hasta ese momento y cuyo fin era viabilizar la salida ordenada del mercado de las empresas en falencia económica. El sistema concursal trajo consigo la posibilidad de una reestructuración y/o recuperación de las empresas, ofreciéndoles una oportunidad importante a los acreedores de decidir reestructurar los pasivos del deudor a tasas de intereses razonables que permitan el reflotamiento de la empresa y la conservación de la unidad productiva, o también la liquidación ordenada de los pasivos y su distribución entre los acreedores, respetando un orden de preferencia establecido en la propia ley. De esta forma se otorgaba una mayor preponderancia a la decisión colegiada, conjunta y mayoritaria de los acreedores frente a la decisión individual del deudor, y sobre la base de la viabilidad económica financiera de la empresa concursada.

La expedición de la LGSC obedeció, en parte, a una serie de problemas que las Comisiones de Procedimientos Concursales no podían afrontar con la legislación previa. Según la exposición de motivos de la ley, los problemas más resaltantes eran estos: (a) procedimientos amplios y muy detallados que impedían la celeridad de tramitación y, por consiguiente, provocaban retraso en la adopción de decisiones por las juntas de acreedores; (b) diversidad de leyes ad hoc en materia concursal que generaban confusión y aumento del costo en la administración del sistema; (c) normas de fiscalización y control insuficientes; (d) inequidades en el tratamiento de la acreencia laboral; (e) intervención inadecuada del Poder Judicial, que obstaculizaba y suspendía las decisiones administrativas y las de las juntas de acreedores.

En efecto, como lo hemos señalado, solo en la década de 1990 se promulgaron normas y significativas modificaciones legislativas que afectaron al sistema concursal por tener un marco normativo no tan uniforme. Así, primero fue la Ley de Reestructuración Empresarial, Ley 26116, de 1992; luego el Decreto Legislativo 845 de 1996; y, posteriormente, la Ley de Fortalecimiento del Sistema de Reestructuración Patrimonial, Ley 27146, de 1999, pero también el procedimiento transitorio en el Decreto de Urgencia 064-99.

Esta última norma, es decir, el Decreto de Urgencia 064-99, obedeció a la saturación y demora de la Comisión de Reestructuración Patrimonial del Indecopi y sus nueve comisiones delegadas en el ámbito nacional (ODI) en la tramitación de los procedimientos de 
insolvencia presentados. Adicionalmente, se buscaba facilitar el acceso a los beneficios que otorga un régimen concursal, descentralizando a los agentes aplicadores del procedimiento transitorio y flexibilizando o simplificando los requisitos para ingresar en él. En consideración a ello, el procedimiento transitorio se crea para (a) descentralizar las funciones del Indecopi, (b) reducir y flexibilizar los requisitos para gozar de los beneficios del sistema concursal, (c) agilizar el reconocimiento de los créditos, (d) controlar mejor la asunción de nuevas deudas por parte de la empresa concursada, (e) propiciar reuniones y, con ello, decisiones más rápidas de las juntas de acreedores, entre otras razones.

Por las razones expuestas, resultó necesario elaborar un marco normativo sólido que pueda perdurar en el futuro como principal fuente del derecho concursal peruano. Así, en el texto original del artículo I de la LGSC se estableció como principal objetivo del sistema concursal:

La permanencia de la unidad productiva, la protección del crédito y el patrimonio de la empresa. Los agentes del mercado procurarán una asignación eficiente de sus recursos durante los procedimientos concursales orientando sus esfuerzos a conseguir el máximo valor del patrimonio en crisis².

Y la norma del artículo II de la misma ley señala:

Los procedimientos concursales tienen por finalidad propiciar un ambiente idóneo para la negociación entre los acreedores y el deudor sometido a concurso, que les permita llegar a un acuerdo de reestructuración o, en su defecto, a la salida ordenada del mercado, bajo reducidos costos de transacción.

La LGSC ha logrado así mantenerse vigente por más de dieciocho años, más allá de naturales modificaciones acordes al desarrollo de los tiempos, lo cual evidencia que dicho cuerpo legislativo ha sido un gran aporte para el ordenamiento jurídico. Y el curso de derecho concursal sigue formando parte del currículo de estudios de la Facultad de Derecho de la Universidad de Lima, estando a cargo del profesor Alfonso Pérez-Bonany.

Por último, no podemos dejar de destacar la importancia que se le dio en la Facultad de Derecho al curso de derecho de seguros, cuyo primer profesor fue el abogado especializado doctor Javier Cavero Egúsquiza, hasta que se retiró por límite de edad el 2012; ahora el profesor del curso es el doctor Américo Hidalgo, abogado y magíster formado en la Universidad de Lima. Como se sabe, el contrato de seguros estuvo regulado en el Código de Comercio de 1902, en sus artículos 375 hasta el 429 inclusive, y actualmente lo está por la Ley 29946, vigente desde el 27 de mayo del 2013; sin embargo, el tratamiento legislativo y comercial del contrato de seguros fue muy especial en los últimos cuarenta años, pues debemos recordar que dicha actividad fue afectada y hasta cierto

2 Este texto fue posteriormente modificado por el Decreto Legislativo 1050 del 2008. 
punto objeto de proyectos de estatización del sistema financiero y de seguros, e inclusive de una Ley de Expropiación de Bancos, Financieras y Seguros. Estas decisiones políticas afectaron la celebración de este tipo de contrato, pero por su importancia comercial no se debía dejar de lado, razón por la cual consideramos que su contenido y alcances debían ser materia de estudios.

Para finalizar la exposición de la activa participación de la Facultad de Derecho de la Universidad de Lima en la legislación comercial peruana, no debemos dejar de destacar la participación de lus et Praxis y de las Asociaciones ADV Editores y Athina, conformada por los alumnos de la Facultad de Derecho de esta casa de estudios, los cuales editan las revistas lus et Praxis (revista oficial de la Facultad de Derecho de la Universidad de Lima), Advocatus y Athina, respectivamente, en las que alumnos y profesores han participado con artículos para dar a conocer sus interpretaciones y críticas de la normativa, lo que resulta un aporte para la doctrina en diversas materias.

Asimismo, se han organizado múltiples eventos, entre en los que podemos destacar dos:

- El Il Congreso Nacional del Derecho del Consumidor, organizado por la Facultad de Derecho de la Universidad de Lima e lus et Praxis (del 14 al 16 de noviembre del 2012), donde participaron, entre otros, los profesores Gustavo Rodríguez, Dante Mendoza Antonioli, Marco Antonio Ortega Piana, Daniel Echaiz Moreno, Julio Baltazar Durand Carrión, Juan José Blossiers Mazzini, Gianina Danziger Castañeda, Alonso Morales Acosta y el suscrito.

- El Conversatorio sobre Arbitraje de Consumo (llevado a cabo el 1 de julio del 2016), organizado por la Facultad de Derecho de la Universidad de Lima, donde participó, entre otros, el doctor Gonzalo García Calderón Moreyra.

Para concluir, debo agradecer de manera especial que se me haya solicitado esta recopilación y a la vez destacar la calidad de un porcentaje importante de profesores de la Facultad de Derecho de la Universidad de Lima, que tienen a su cargo los cursos de las áreas de derecho comercial a las que nos hemos referido, profesores que han estudiado y se han graduado en la misma facultad.

Lima, 8 de noviembre del 2020

\section{REFERENCIAS}

Beaumont Callirgos, R., y Castellares Aguilar, R. (2000). Comentarios a la nueva Ley de Títulos Valores. Análisis artículo por artículo. Gaceta Jurídica.

Decreto Legislativo 788 de 1994. Declaran en reorganización al Instituto Nacional de Defensa de la Competencia y de la Protección de la Propiedad Intelectual-Indecopi. 
31 de diciembre de 1994. https://www.leyes.congreso.gob.pe/Documentos /DecretosLegislativos/00788.pdf

Ley 26887 de 1997. Ley General de Sociedades. 5 de diciembre de 1997. https://docs. peru.justia.com/federales/leyes/26887-dec-5-1997.pdf

Ley 27809 del 2002. Ley General del Sistema Concursal. 5 de agosto del 2002. http://www. osterlingfirm.com/Documentos/cdi/Formatos_new/NORMAS_REGISTRALES/ LEYES_Y_DECRETOS_LEYES/Ley_27809.pdf

Nuevo derecho societario. I Seminario Nacional sobre la Ley General de Sociedades. (1998). Universidad de Lima, Fondo de Desarrollo Editorial. 